\title{
PAX stains in hematologic malignancies, a diagnostic pitfall: a comparative study evaluating monoclonal PAX8s, polyclonal PAX2, and PAX5
}

\author{
Joanna L. Conant ${ }^{1} \cdot$ Michael DeSarno $^{2} \cdot$ Abiy B. Ambaye $^{1} \cdot$ Ronald Bryant $^{1}$. \\ Maryam Zenali ${ }^{1}$
}

Received: 8 June 2015 / Accepted: 18 January 2016 /Published online: 26 January 2016

(C) Springer-Verlag Berlin Heidelberg 2016

\begin{abstract}
Paired-box (PAX) genes constitute a family of genes involved in development. PAX5 is expressed in B lineage lymphomas. Recent studies report positive staining of polyclonal PAX8 in B cell proliferations due to N-terminus homology between PAX5 and PAX8. However, the expression of PAX2 or monoclonal antibodies against the $\mathrm{N}$-terminus of PAX8 (PAX8N) in these neoplasms has not been fully evaluated. The goal of this study was to evaluate the expression of PAX8N, monoclonal C-terminus PAX8 (PAX8C), and polyclonal PAX2 in hematologic malignancies and to compare the results with PAX5. Immunohistochemical (IHC) staining for PAX5, PAX8N, PAX8C, and PAX2 was performed on follicular lymphoma grades 1-3 (FL1-FL3), diffuse large B cell lymphoma (DLBCL), small lymphocytic lymphoma (SLL), nodal marginal zone lymphoma (MZL), mantle cell lymphoma (MCL), classical Hodgkin lymphoma (cHL), plasmacytoma, anaplastic large cell lymphoma (ALCL), and peripheral T cell lymphoma (PTCL). Expression was scored based on percentage of nuclear staining in neoplastic cells. PAX8N staining correlated with PAX5 and was positive in all B cell neoplasms examined, while PAX8C was negative. PAX2 had sporadic expression in FL2, FL3, DLBCL, and MCL. All PAX stains were nearly or entirely negative in plasmacytomas, ALCL, and PTCL. PAX8N stains B cell neoplasms and its positivity parallels
\end{abstract}

Joanna L. Conant

joanna.conant@uvmhealth.org

1 Department of Pathology and Laboratory Medicine, University of Vermont Medical Center, 111 Colchester Ave, Burlington, VT 05401, USA

2 Medical Biostatistics Department, University of Vermont, Burlington, VT, USA
PAX5. PAX8C does not stain any of the aforementioned neoplasms, and PAX2 shows no distinct pattern. While PAX8C has a role in discriminating lymphoid and epithelial malignancies, PAX8N, polyclonal PAX8, and PAX2 do not. This study illustrates the importance of knowing specific IHC targets to avoid misdiagnosis.

Keywords B cell lymphomas · Immunoperoxidase · Polyclonal PAX8 $\cdot$ Monoclonal PAX8-N $\cdot$ Monoclonal PAX8-C $\cdot$ PAX2

Paired-box (PAX) genes are a family of transcription factors that are involved in embryonic development and differentiation. Their expression persists in a subset of mature tissue such as renal tubules, while in many others disappears. PAX protein expression may resurface during the development of certain malignancies $[1,2]$. PAX genes can be classified into four subgroups based on their gene sequence; embryonic expression is also correlated within these subgroups. PAX2, PAX5, and PAX8 are categorized together under one of these four classes [3]. PAX5 is involved in early B lymphocyte differentiation and is expressed in neoplastic B lymphocytes as well as all stages of benign B lymphocytes except for plasma cells $[4,5]$; PAX8 is associated with tumors arising from the thyroid, kidney, and upper urinary tract and the Müllerian system; PAX2 is expressed in Müllerian neoplasms and subsets of kidney tumors [1-3].

Few recent studies report expression of polyclonal PAX8 by non-neoplastic lymphoid cells using immunohistochemistry (IHC) [6-8]. However, structural studies have led to the hypothesis that positive staining of polyclonal PAX8 in some lymphoid neoplasms is due to cross reactivity with the $\mathrm{N}$-terminal portion of the PAX5 protein. Upon gene sequence analysis, the N-terminus of PAX5 and PAX8 contained high 
sequence homology [6]. There have also been scattered reports of expression of PAX2 in benign lymphocytes and lymphoid neoplasms, though the possibility of cross reactivity has also been raised $[9,10]$.

Index case A 66-year-old woman presented to our institution with abdominal pain and was found to have an $8-\mathrm{cm}$ right kidney mass and enlarged retroperitoneal lymph nodes by imaging, which was suspicious for renal cell carcinoma. Biopsy of the mass contained a spindle cell neoplasm with marked pleomorphism and hyperchromasia. The neoplastic cells were negative for keratin AE1-AE3 IHC staining but positive for PAX8, and the case was signed out as undifferentiated malignant neoplasm suggestive of sarcomatoid renal cell carcinoma (Fig. 1). She then underwent a total nephrectomy. The kidney contained a large mass with discohesive cells exhibiting highly pleomorphic and anaplastic nuclei, frequent apoptosis, and numerous mitoses. Again, the keratin AE1-AE3 was negative and PAX8 was positive (Fig. 2). Given the morphology, a leucocyte common antigen (LCA; CD45) IHC was performed and was positive. Additional confirmatory stains including CD20 were positive, and the case was signed out as a diffuse large B cell lymphoma (DLBCL).

Upon identification of monoclonal PAX8 staining in the aforementioned DLBCL, additional cases were further evaluated for staining with the antibody for quality assurance purposes, and all demonstrated diffuse and strong nuclear staining with monoclonal PAX8. The monocloncal PAX8 clone used in these cases (mouse monoclonal, clone MRQ-50, Ventana, ready to use) is targeted at the N-terminus. In our review of the literature, there have been no reports of monoclonal PAX8 antibody expression in lymphoid tissue; positive IHC staining has only been reported with polyclonal PAX8 antibodies [6-8].

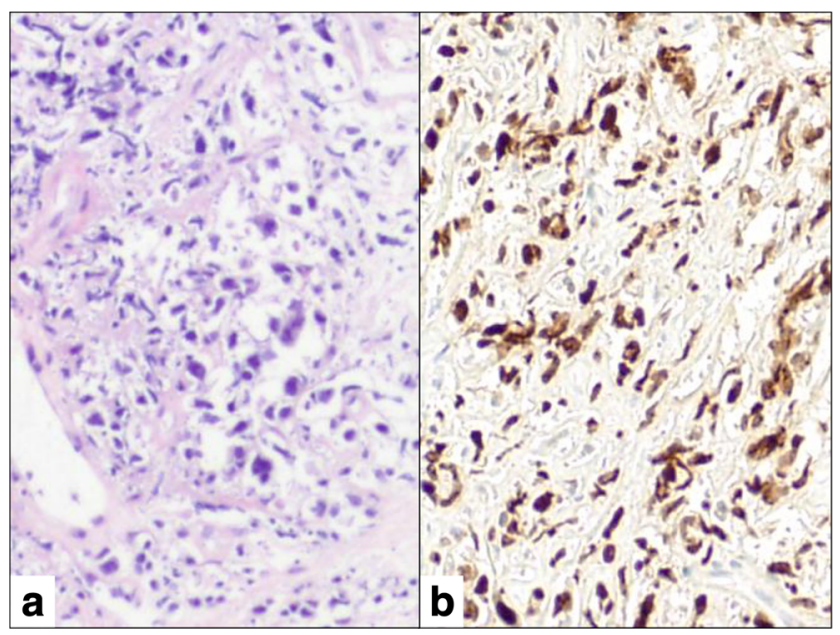

Fig. 1 Kidney biopsy from index case. a Hematoxylin and eosin. b PAX8N (original magnification $\times 100[\mathbf{a}, \mathbf{b}]$ )
Therefore, we sought to evaluate the expression of two monoclonal PAX8 antibodies-one directed at the $\mathrm{N}$-terminus $(\mathrm{PAX} 8 \mathrm{~N})$ and one directed at the $\mathrm{C}$-terminus (PAX8C) - in a larger subset of hematologic malignancies. In parallel, since PAX2 expression has not been fully evaluated in different hematologic neoplasms, we studied the IHC staining pattern of PAX2 in the same cohort of tumors. The goal of this study was to determine the likelihood of immunoreactivity for monoclonal PAX8 (N-terminus vs. C-terminus) and PAX2 in subsets of lymphoproliferative disorders and to compare these staining profiles to that of PAX5.

\section{Materials and methods}

\section{Patients and samples}

After the study was approved by the University of Vermont Institutional Review Board and the Vermont Cancer Center Protocol Review and Monitoring Committee, a laboratory information system search was conducted from the University of Vermont Medical Center archives for cases of follicular lymphoma grades 1-3 (FL1-3), diffuse large B cell lymphoma (DLBCL), small lymphocytic lymphoma (SLL), nodal marginal zone lymphoma (MZL), mantle cell lymphoma (MCL), classical Hodgkin lymphoma (cHL), plasmacytoma, anaplastic large cell lymphoma (ALCL), and peripheral T cell lymphoma (PTCL) specimens between 2001 and 2011. Cases were reviewed and selected for inclusion in the study if the diagnosis was confirmed and adequate tissue was available for further IHC studies. A total of 20 cases of FL1, 13 cases of FL2, 10 cases of FL3, 23 cases of DLBCL, 23 cases of SLL, 8 cases of MZL, 9 cases of MCL, 15 cases of cHL, 14 cases of plasmacytoma, 8 cases of ALCL (4 ALK-positive and 4 ALKnegative), and 4 cases of PTCL were included in the study.

\section{Immunohistochemical staining}

Upon review of all cases, a formalin-fixed, paraffin-embedded tissue block was selected from each case for IHC study. For PAX5, PAX8C, and PAX2, 5- $\mu$ m-thick tissue sections were cut, deparaffinized in xylene, rehydrated through a graded series of ethanol solutions, and then washed with distilled water. The slides were then subjected to IHC staining for PAX5 (rabbit monoclonal, clone SP34, Thermo Scientific, 1:50 dilution, $8.0 \mathrm{pH}$ EDTA retrieval), PAX8C (mouse monoclonal, clone PAX8R1, Abcam, 1:50 dilution, $6.0 \mathrm{pH}$ citrate retrieval), and PAX2 (rabbit polyclonal, clone 08-1483, Invitrogen, 1:50 dilution, $6.0 \mathrm{pH}$ citrate retrieval) using the UV LP Thermo Scientific IHC detection kit and stained using the Lab Vision auto stainer. For PAX8N (mouse monoclonal, clone MRQ-50, Ventana, ready to use), 5- $\mu \mathrm{m}$-thick tissue sections were cut, heated, then placed on the fully automated 
Fig. 2 Kidney resection from index case. a Hematoxylin and eosin. b PAX8N. c PAX8C (original magnification $\times 100$ $[\mathbf{a}, \mathbf{b}])$

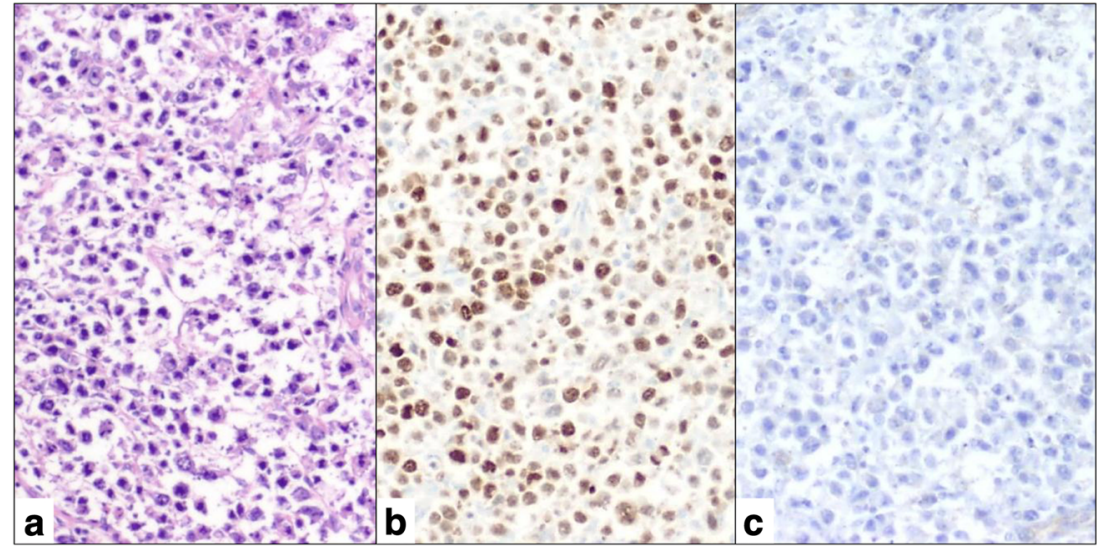

Ventana Benchmark Ultra system using the Opti View IHC detection kit. Positive and negative controls yielded appropriate results. PAX expression was evaluated by two of the authors (JLC and MZ) independently. The few cases with discrepancies in degree of positive scoring were further discussed, and consensus was achieved. Expression for all entities except for cHL was scored based on percentage of nuclear staining: $0=<5 \%, 1=5-25 \%, 2=26-50 \%, 3=51-$ $75 \%$, and $4=>75 \%$. Due to the paucity of Reed-Sternberg cells in a number of cases of cHL, expression was noted to be positive or negative. The intensity of nuclear staining (strong, moderate, weak) was also noted.

\section{Statistical analysis}

In order to compare the IHC expression data for PAX2, PAX8N, and PAX8C to that of PAX5, within individual cases, the raw expression data of all entities except for $\mathrm{cHL}$ was analyzed using the signed rank test, which is the nonparametric equivalent of the paired $t$ test. For $\mathrm{cHL}$, the raw expression data was analyzed using the McNemar's test for agreement. The significance level for all statistical tests was set, a priori, to alpha $=0.05$. The statistical analysis software used for the analyses was SAS, ver. 9.2 (SAS Institute, Inc., Cary, NC, USA).

\section{Results}

Table 1 lists the frequency of scores (0-4) for PAX5, PAX8N, PAX8C, and PAX2 for each of the lymphoid neoplasms examined. In all lymphoid malignancies examined, there was no difference in staining between PAX8N and PAX5 and the expression correlated (Table 2) and was diffusely and strongly positive in FL1-3, DLBCL, SLL, MZL, and, MCL. The Reed-Sternberg cells of cHL were weakly positive for both PAX5 and PAX8N, though the staining of PAX8N was weaker than that of PAX5. PAX8C expression was negative in all neoplasms examined. PAX2 showed some expression in FL2, FL3, DLBCL, and MCL, but was usually weak or moderate in intensity. All PAX stains were nearly or entirely negative in plasmacytomas, ALCL, and PTCL.

\section{Discussion}

It is known that PAX5 immunoproxidase is a robust marker of B cell lymphoid proliferations. In contrast, in the same subset of tumors, expression status of other PAX markers such as PAX8 is not widely recognized. PAX5 is involved in early B lymphocyte differentiation and is well known to be expressed in B cell neoplasms as well as all stages of benign B lymphocytes except for plasma cells [4, 5]. Per Adams et al [11], while CD20 and CD79a are superior, in mature lymphoid neoplasms PAX5 should be considered as a third-line B cell marker in equivocal cases. In their review, which included a host of B cell and T cell neoplasms, they identified a specificity of $88 \%$ and sensitivity of $82 \%$ for PAX5 in detecting mature B cell neoplasms.

In parallel, a study by Torlakovic et al [5] showed that $100 \%$ of all FL, SLL, MZL, and MCL and $90 \%$ of DLBCL were strongly immunoreactive for PAX5, with an additional $6 \%$ of DLBCL with weak immunoreactivity. The Reed-Sternberg cells in cHL were weakly positive for PAX5 (92\%) and another $5 \%$ were strongly positive for PAX5. All (100\%) of ALCL and PTCL examined were negative for PAX5. Only $5 \%$ (2/39) of plasma cell myelomas had weak positivity for PAX5, while the remainder of myelomas as well as the few examined plasmacytomas were negative. The result of PAX 5 staining in our study closely parallels these findings, with $100 \%$ of FL, DLBCL, SLL, MZL, and MCL being strongly positive for PAX5 and $100 \%$ of cHL being weakly positive for PAX5. Of the plasmacytomas, $21 \%$ (3 of 14) were positive for PAX5 and $100 \%$ of ALCL and PTCL were negative. 
Table 1 Frequency of PAX expression by score in lymphoid neoplasms

\begin{tabular}{|c|c|c|c|c|c|c|c|c|c|}
\hline & PAX5 & PAX8N & PAX8C & PAX2 & & PAX5 & PAX8N & PAX8C & PAX2 \\
\hline \multicolumn{5}{|l|}{ FL1, $n=20$} & \multicolumn{5}{|l|}{ MZL, $n=8$} \\
\hline $0:<5 \%$ & 0 & 0 & 20 & 20 & $0:<5 \%$ & 0 & 0 & 8 & 7 \\
\hline 1: $5-25 \%$ & 0 & 0 & 0 & 0 & 1: $5-25 \%$ & 0 & 0 & 0 & 0 \\
\hline $2: 26-50 \%$ & 0 & 0 & 0 & 0 & $2: 26-50 \%$ & 0 & 1 & 0 & 1 \\
\hline 3: $51-75 \%$ & 1 & 3 & 0 & 0 & 3: 51-75\% & 0 & 1 & 0 & 0 \\
\hline $4:>75 \%$ & 19 & 17 & 0 & 0 & $4:>75 \%$ & 8 & 6 & 0 & 0 \\
\hline \multicolumn{5}{|l|}{ FL2, $n=13$} & \multicolumn{5}{|l|}{ MCL, $n=9$} \\
\hline $0:<5 \%$ & 0 & 0 & 13 & 10 & $0:<5 \%$ & 0 & 0 & 9 & 2 \\
\hline 1: $5-25 \%$ & 0 & 0 & 0 & 3 & 1: $5-25 \%$ & 0 & 0 & 0 & 2 \\
\hline $2: 26-50 \%$ & 0 & 0 & 0 & 0 & $2: 26-50 \%$ & 0 & 0 & 0 & 3 \\
\hline 3: $51-75 \%$ & 0 & 1 & 0 & 0 & $3: 51-75 \%$ & 0 & 0 & 0 & 2 \\
\hline $4:>75 \%$ & 13 & 12 & 0 & 0 & $4:>75 \%$ & 9 & 9 & 0 & 0 \\
\hline \multicolumn{5}{|l|}{ FL3, $n=10$} & \multicolumn{5}{|c|}{ Plasmacytoma, $n=14$} \\
\hline $0:<5 \%$ & 0 & 0 & 13 & 0 & $0:<5 \%$ & 11 & 12 & 14 & 14 \\
\hline 1: $5-25 \%$ & 0 & 0 & 0 & 0 & $1: 5-25 \%$ & 1 & 0 & 0 & 0 \\
\hline 2: $26-50 \%$ & 0 & 0 & 0 & 1 & $2: 26-50 \%$ & 0 & 0 & 0 & 0 \\
\hline 3: $51-75 \%$ & 0 & 1 & 0 & 8 & $3: 51-75 \%$ & 0 & 0 & 0 & 0 \\
\hline $4:>75 \%$ & 13 & 12 & 0 & 1 & $4:>75 \%$ & 2 & 2 & 0 & 0 \\
\hline \multicolumn{5}{|c|}{ DLBCL, $n=23$} & \multicolumn{5}{|l|}{ ALCL, $n=8$} \\
\hline $0:<5 \%$ & 0 & 0 & 23 & 5 & $0:<5 \%$ & 8 & 8 & 8 & 8 \\
\hline 1: $5-25 \%$ & 0 & 0 & 0 & 4 & $1: 5-25 \%$ & 0 & 0 & 0 & 0 \\
\hline $2: 26-50 \%$ & 0 & 1 & 0 & 4 & $2: 26-50 \%$ & 0 & 0 & 0 & 0 \\
\hline 3: $51-75 \%$ & 0 & 1 & 0 & 9 & $3: 51-75 \%$ & 0 & 0 & 0 & 0 \\
\hline $4:>75 \%$ & 23 & 21 & 0 & 1 & $4:>75 \%$ & 0 & 0 & 0 & 0 \\
\hline \multicolumn{5}{|l|}{ SLL, $n=23$} & \multicolumn{5}{|l|}{ PTCL, $n=4$} \\
\hline $0:<5 \%$ & 0 & 0 & 23 & 23 & $0:<5 \%$ & 4 & 4 & 4 & 4 \\
\hline $1: 5-25 \%$ & 0 & 0 & 0 & 0 & $1: 5-25 \%$ & 0 & 0 & 0 & 0 \\
\hline $2: 26-50 \%$ & 0 & 1 & 0 & 0 & $2: 26-50 \%$ & 0 & 0 & 0 & 0 \\
\hline 3: $51-75 \%$ & 7 & 1 & 0 & 0 & $3: 51-75 \%$ & 0 & 0 & 0 & 0 \\
\hline $4:>75 \%$ & 16 & 21 & 0 & 0 & $4:>75 \%$ & 0 & 0 & 0 & 0 \\
\hline \multicolumn{10}{|l|}{ cHL, $n=15$} \\
\hline Positive & 15 & 12 & 0 & 1 & & & & & \\
\hline
\end{tabular}

Table 2 Comparison of PAX5 vs PAX8N, PAX8C, and PAX2 ( $p$ values)

\begin{tabular}{llll}
\hline & $\begin{array}{l}\text { PAX5 vs } \\
\text { PAX8N }\end{array}$ & $\begin{array}{l}\text { PAX5 vs } \\
\text { PAX8C }\end{array}$ & $\begin{array}{l}\text { PAX5 vs } \\
\text { PAX2 }\end{array}$ \\
\hline FL1 $(n=20)$ & 0.63 & $<0.0001$ & $<0.0001$ \\
FL2 $(n=13)$ & 1.0 & 0.0002 & 0.0002 \\
FL3 $(n=10)$ & 1.0 & $<0.0001$ & 0.004 \\
DLBCL $(n=23)$ & 0.5 & $<0.0001$ & $<0.0001$ \\
SLL $(n=23)$ & 0.29 & $<0.0001$ & $<0.0001$ \\
MZL $(n=8)$ & 0.5 & 0.01 & 0.01 \\
MCL $(n=9)$ & 1.0 & 0.004 & 0.004 \\
cHL $(n=15)$ & 0.25 & $<0.0001$ & 0.0001 \\
Plasmacytoma $(n=14)$ & 1.0 & 0.25 & 0.25 \\
ALCL $(n=8)$ & 1.0 & 1.0 & 1.0 \\
PTCL $(n=4)$ & 1.0 & 1.0 & 1.0 \\
\hline
\end{tabular}

Several studies have reported PAX8 positivity in B cell neoplasms, but this immunoreactivity is theorized to be due to cross-reactivity with PAX5 [6-8]. Moretti et al [6] demonstrated that there is high gene sequence homology between the N-terminal portions of PAX8 and PAX5, confirming the theory of PAX8 positivity due to cross reactivity. These studies reporting PAX8 immunoreactivity in B cell neoplasms have all used polyclonal PAX8 IHC stains.

Morgan et al [7] demonstrated that polyclonal PAX8 was positive in a variety of lymphoid neoplasms and that the staining paralleled that of PAX5. Their study also indicated that monoclonal PAX8 was negative in the same neoplasms. Their monoclonal PAX8 antibody was the same antibody used in our study for PAX8C. Our findings confirmed that the monoclonal PAX8 antibody directed at the $\mathrm{C}$-terminus are negative in lymphoid neoplasms. 
Fig. 3 Example of PAX staining in SLL. a PAX5. b PAX8N. c PAX8C (original magnification $\times 100[\mathbf{a}-\mathbf{c}])$

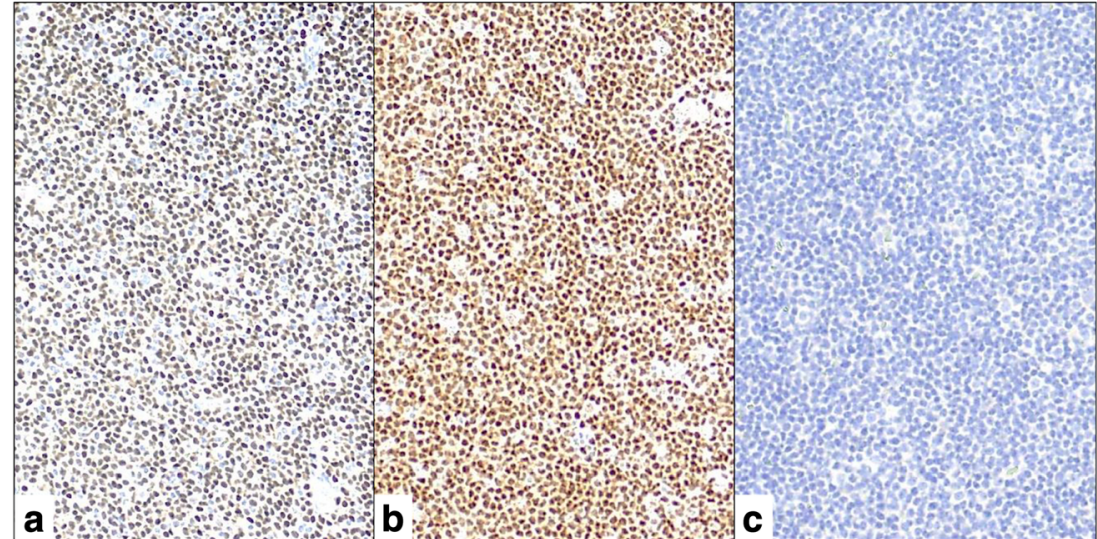

To the best of our knowledge, there have been no published reports on the cross reactivity of monoclonal PAX8 antibodies that are directed at the $\mathrm{N}$-terminus. In this study, we compared two monoclonal PAX8 antibodies, one directed at the $\mathrm{N}$-terminus and one directed at the C-terminus, in a variety of lymphoid neoplasms known to be either PAX5 positive (FL1-3, DLBCL, SLL, MZL, MCL, cHL) or PAX5 negative (plasmacytomas, ALCL, and PTCL).

We found that PAX8N is immunoreactive in FL1-3, DLBCL, SLL, MZL, and MCL, and its immunoreactivity paralleled that of PAX5; both exhibited diffuse and strong nuclear staining (Fig. 3). Both were also positive in cHL, albeit weaker than the other B cell neoplasms. PAX8C, however, was not immunoreactive in any of the aforementioned neoplasms. PAX5, PAX8N, and PAX8C IHC stains were nearly or entirely negative in plasmacytomas, ALCL, and PTCL. This is not unexpected given that these entities do not typically express PAX5. There have been some reports of PAX5 positivity in some ALK-negative ALCL, but we did not see any PAX5 staining at all $[12,13]$.

These findings support previous studies that suggest PAX8 immunoreactivity in B cell neoplasms is due to cross reactivity with the N-terminus of PAX5 [6-8]. These studies have all demonstrated positive staining with polyclonal PAX8 antibodies; ours is the first to demonstrate positive staining with a monoclonal PAX8 antibody. Of note, subsequent staining in our index case of renal DLBCL resulted in negative staining for PAX8C (Fig. 2).

We noted a variable degree of PAX2 expression in FL2, FL3, DLBCL, and MCL, in no distinct pattern and generally weak or moderate in intensity. While this spurious staining may be due to cross reactivity, the observed staining pattern is not as uniformly predictable as that of PAX8N. The variability seen with PAX2 may be due to the use of a polyclonal antibody. The significance or utility of PAX2 in lymphoid malignancies remains unknown, but it is unlikely to play a key role in diagnosis.
This study illustrates the importance of knowing the target of IHC stains when evaluating expression in order to avoid confusion and misdiagnosis. While PAX8C may have a role in discriminating between lymphoid and epithelial malignancies, neither PAX8N nor polyclonal PAX8 is discriminating. A small follow-up validation study at our institution (not reported) showed PAX8C to be less sensitive than PAX8N in detecting renal and Müllerian malignancies. Therefore, we recommend continuing to use PAX8N in clinical practice with knowledge that B lymphocytes, both benign and neoplastic, will be immunoreactive. With this knowledge, lymphoid tissue in tissue sections can be used as an internal control.

\section{Compliance with ethical standards}

Funding No funding was provided for this study.

Ethical approval All procedures performed in studies involving human participants were in accordance with the ethical standards of the institutional and/or national research committee and with the 1964 Helsinki Declaration and its later amendments or comparable ethical standards.

Conflict of interest The authors declare that they have no competing interests.

\section{References}

1. Muratovska A, Zhou C, He S, Goodyer P, Eccles MR (2003) Paired-box genes are frequently expressed in cancer and often required for cancer cell survival. Oncogene 22(39):7989-97

2. Mansouri A, Hallonet M, Gruss P (1996) Pax genes and their roles in cell differentiation and development. Curr Opin Cell Biol 8(6): $851-7$

3. Chi N, Epstein JA (2002) Getting your Pax straight: Pax proteins in development and disease. Trends Genet 18(1):41-7 
4. Desouki MM, Post GR, Cherry D, Lazarchick J (2010) PAX-5: a valuable immunohistochemical marker in the differential diagnosis of lymphoid neoplasms. Clin Med Res 8(2):84-8

5. Torlakovic E, Torlakovic G, Nguyen PL, Brunning RD, Delabie J (2002) The value of anti-pax-5 immunostaining in routinely fixed and paraffin-embedded sections: a novel pan pre-B and B-cell marker. Am J Surg Pathol 26(10):1343-50

6. Moretti L, Medeiros LJ, Kunkalla K, Williams MD, Singh RR, Vega F (2012) N-terminal PAX8 polyclonal antibody shows cross-reactivity with $\mathrm{N}$-terminal region of PAX5 and is responsible for reports of PAX8 positivity in malignant lymphomas. Mod Pathol 25(2):231-6

7. Morgan EA, Pozdnyakova O, Nascimento AF, Hirsch MS (2013) PAX8 and PAX5 are differentially expressed in B-cell and T-cell lymphomas. Histopathology 62(3):406-13

8. Ozcan A, Shen SS, Hamilton C et al (2011) PAX 8 expression in non-neoplastic tissues, primary tumors, and metastatic tumors: a comprehensive immunohistochemical study. Mod Pathol 24(6): 751-64

9. Ordóñez NG (2012) Value of PAX2 immunostaining in tumor diagnosis: a review and update. Adv Anat Pathol 19(6):401-9

10. Gilmore L, Dewar R (2011) Caution in metastatic renal cell carcinoma within lymph nodes: PAX-2 expression is also seen in nodal lymphocytes. Arch Pathol Lab Med 135(4):414

11. Adams H, Liebisch P, Schmid P, Dirnhofer S, Tzankov A (2009) Diagnostic utility of the B-cell lineage markers CD20, CD79a, PAX5, and CD19 in paraffin-embedded tissues from lymphoid neoplasms. Appl Immunohistochem Mol Morphol 17(2):96-101

12. Ong DM, Cummins KD, Pham A, Grigoriadis G (2015) PAX5expressing ALK-negative anaplastic large cell lymphoma with extensive extranodal and nodal involvement. BMJ Case Rep. 2015

13. Hagiwara M, Tomita A, Takata K et al (2012) Primary cutaneous CD30 positive T-cell lymphoproliferative disorders with aberrant expression of PAX5: report of three cases. Pathol Int 62(4):264-70 